\title{
Hyperglycemia Is a Strong Prognostic Factor of Lethality in Methanol Poisoning
}

\author{
Hossein Sanaei-Zadeh • Shahrooz Kazemi Esfeh • \\ Nasim Zamani • Farkhondeh Jamshidi • Shahin Shadnia
}

Published online: 20 February 2011

(C) American College of Medical Toxicology 2011

\begin{abstract}
Methanol poisoning is seen in the form of isolated episodes, or intentional ingestion and epidemics. Despite its efficient treatment, methanol poisoning has high morbidity and mortality rates. So far, several studies have been performed to identify the prognostic factors in methanol poisoning. Recently, during the treatment of patients with methanol poisoning, we observed that patients' blood glucose levels were high on presentation to the hospital, particularly in those who expired. Through a literature search, we found that no studies have been performed on blood glucose levels or hyperglycemia in methanol poisoning. Therefore, the present retrospective study was done as a preliminary investigation to understand whether there was a meaningful relationship between methanol poisoning and blood glucose level on
\end{abstract}

H. Sanaei-Zadeh $\cdot$ N. Zamani $\cdot$ F. Jamshidi

Department of Forensic Medicine and Toxicology,

Tehran University of Medical Sciences,

Tehran, Iran

\section{S. Kazemi Esfeh}

Department of Forensic Medicine and Clinical Toxicology,

Faculty of Medicine, Shahid Beheshti University of Medical Sciences,

Tehran, Iran

\section{S. Shadnia}

Clinical Toxicology Department, Loghman-Hakim Hospital Poison

Center, Faculty of Medicine and Toxicological Research Center,

Shahid Beheshti University of Medical Sciences,

Tehran, Iran

H. Sanaei-Zadeh $(\square)$

Department of Forensic Medicine and Toxicology,

Tehran University of Medical Sciences,

Hazrat Rasoul Akram Hospital,

Niayesh Street, Sattar-Khan Ave,

1445613131, Tehran, Iran

e-mail: sanaeizadeh@gmail.com presentation, and also if hyperglycemia could be considered as a prognostic factor for mortality. In this retrospective study, a review of the hospital charts was performed for all patients who were treated for methanol poisoning from March 2003 to March 2010 in two hospitals in Tehran, Iran. Those with definitive diagnosis of methanol poisoning, no history of diabetes mellitus, and normal or low body mass index $(<25)$ were included. Patients' demographic information, clinical manifestations, time elapsed between ingestion and presentation, blood glucose level on presentation (before treatment), results of arterial blood gas analysis, and the clinical outcome were recorded. Statistical analysis was done using SPSS software (version 17, Chicago, Illinois, USA) and application of Mann-Whitney $U$ test, Pearson's chi-square test, Pearson correlation coefficient $(r)$, receiver operating characteristic (ROC) curve, and logistic regression. $P$ values less than 0.05 were considered as the statistically significant levels. Ninetyfive patients with methanol poisoning met the inclusion criteria and were included in the study. Of these, 91 $(96 \%)$ were male and $4(4 \%)$ were female. Mean age was $31.61 \pm 14.3$ years (range, 13 to 75 ). Among the 95 patients, 68 survived (72\%) and 27 expired (28\%). Median blood glucose level was $144 \mathrm{mg} / \mathrm{dL}$ (range, 75 to 500). There was no significant statistical correlation between blood glucose level and time of treatment, age, $\mathrm{pCO}_{2}$, or serum bicarbonate concentration, but blood glucose level had a statistically significant correlation with $\mathrm{pH}(r=-0.242$, $P=0.02)$ and base deficit $(r=0.230, P=0.03)$. The mean blood glucose level was $140 \pm 55$ and $219 \pm 99 \mathrm{mg} / \mathrm{dL}$ in the survivor and non-survivor patients, respectively $(P<001)$. Considering the cutoff level of $140 \mathrm{mg} / \mathrm{dL}$ for blood glucose and using logistic regression analysis, and adjusting according to the admission data with significant statistical difference in the two study groups, the odds ratio for hyperglycemia as a risk factor for death was 6.5 
( $95 \%$ confidence interval $=1.59-26.4$ ). Our study showed that blood glucose levels were high in methanol poisoning and even higher in those who died in comparison with the survivors. Therefore, hyperglycemia might be a new prognostic factor in methanol poisoning, but further studies are needed to determine whether controlling hyperglycemia has therapeutic consequences.

Keywords Methanol poisoning · Prognosis . Hyperglycemia $\cdot$ Mortality

\section{Introduction}

Methanol is converted to its toxic metabolite, formic acid, which is responsible for the adverse effects seen in methanol poisoning $[1,2]$. In the early stage of methanol poisoning, toxic effects are caused by the metabolic acidosis, due to formation of formic acid [3]. In the late stage of poisoning, as formate accumulates, toxicity is mainly due to acidosis and the histotoxic effects of formate that prevents the mitochondrial respiration (tissue hypoxia) [4]. Therefore, the production of lactate causes increased acidosis, and the acidosis shifts the equilibrium between formate and formic acid, favoring the protonated formic acid, causing increased cellular penetration of the ionized form $[2,5]$. In this late stage, metabolic acidosis is usually a result of the combination of formate accumulation and lactic acidosis [6, 7]. Early clinical features of poisoning are headache, vertigo, nausea, vomiting, and abdominal pain, but these are also seen in later stages. Visual disturbances may appear alone or with other symptoms [1, 6, 8, 9]. Later in the course, severely poisoned patients may refer with coma, seizure, blindness, gastrointestinal hemorrhage, putaminal hemorrhage and infarcts, and pancreatitis [1, 6, 10-12].

Specific treatment of methanol poisoning includes intravenous sodium bicarbonate to correct metabolic acidosis $[13,14]$ and antidotal therapy with ethanol or fomepizole to prevent metabolism of methanol to formate $[1,15,16]$. Hemodialysis is also performed for removing methanol and formate to correct metabolic acidosis [1, 2, 16-19]. Administration of folic or folinic acid may have a role in enhancing the metabolism of formate into $\mathrm{CO}_{2}$ and water $[1,20]$.

Methanol poisoning is seen in the form of isolated episodes, or intentional ingestion and epidemics. Mass poisoning with methanol is rare but regularly occurs in developed and developing countries [10, 21-25]. In Iran, alcohol consumption is prohibited, but consumers illegally use the substances like hand-made liquor, smuggled alcohol, industrial alcohol (containing a mixture of varying percentages of ethanol and methanol with color additives), or the pharmaceutical preparations of ethanol that have medical usage. This is the main reason why methanol poisoning is observed in our country in the form of isolated episodes or sometimes in the form of epidemics.

Despite its efficient treatment, methanol poisoning has a high morbidity and mortality rate $[1,10,21-25]$. To date, several studies have been performed to identify the prognostic factors in methanol poisoning [8, 10, 21-26] (Table 1). During the treatment of patients with methanol poisoning, we observed that their blood glucose levels on presentation to the hospital were high, particularly in those who died. No literature was found describing the relationship between blood glucose level and methanol poisoning. We, therefore, performed a retrospective study to evaluate the potential relationship between methanol poisoning and hyperglycemia, as well as to look for potential prognostic elements of this simple test.

\section{Material and methods}

This was performed as a retrospective review of the hospital charts of all patients who had been treated for methanol poisoning from March 2003 to March 2010 in two hospitals in Tehran, Iran (Loghman Hakim and Hazrat Rasul Akram hospitals). Treatment of patients included administration of sodium bicarbonate, ethanol, folic, or folinic acid, and hemodialysis if necessary. Fomepizole is not available in Iran.

Cases with non-definitive diagnosis of methanol poisoning, history of diabetes mellitus (DM), and obesity or overweight (based on calculated body mass index; BMI $>25$ ) were excluded. Definitive diagnosis of poisoning was based on a positive history of alcohol consumption (hand-made, smuggled, industrial), clinical manifestations, result of qualitative and quantitative tests, metabolic acidosis with an increased anion gap, and reports of the computed tomography scan of the brain.

Where the hospital charts were found incomplete in regards to DM or BMI, the patients or their relatives were contacted by phone. In Iran, all deceased patients who have died from any type of poisoning are sent to the forensic medical center (in Tehran: Kahrizak) for autopsy. Review of the autopsy report was performed to add necessary information on DM and BMI. The poisoned patients referred from other medical centers after initial management were excluded from the study if the information on presentation at the primary centers was not available in their files. Also, where the file information was incomplete and contact through telephone or investigation of files and autopsy reports was not possible, the patients were excluded.

Inclusion criteria of the study consisted of definitive diagnosis of methanol poisoning, no history of DM, and 
Table 1 Published articles related to the prognostic factors in methanol poisoning

\begin{tabular}{|c|c|c|c|}
\hline $\begin{array}{l}\text { Published } \\
\text { article (year) }\end{array}$ & Author(s) & $\begin{array}{l}\text { Number of } \\
\text { patients studied }\end{array}$ & $\begin{array}{l}\text { Prognostic factors for lethality or permanent } \\
\text { sequelae }\end{array}$ \\
\hline 1953 & Bennet Jr IL & 323 & Low serum bicarbonate levels [10] \\
\hline 1979 & Naraqi S, et al. & 28 & $\begin{array}{l}\text { Coma or seizure on presentation and prolonged } \\
\text { acidosis [21] }\end{array}$ \\
\hline 1981 & Swartz RD, et al. & 44 & $\begin{array}{l}\text { Coma on presentation and high serum methanol } \\
\text { concentration [26] }\end{array}$ \\
\hline 1989 & Mathieu P, et al. & 10 & $\begin{array}{l}>10 \mathrm{~h} \text { of delay from intake to treatment and } \\
\text { blood formate level }>0.5 \mathrm{~g} / \mathrm{L}[22]\end{array}$ \\
\hline 1998 & Liu JJ, et al. & 50 & $\begin{array}{l}\text { Coma or seizure on presentation, severe metabolic } \\
\text { acidosis, in particular initial arterial } \mathrm{pH}<7 \text { and } \\
\text { high serum methanol concentration }[23]\end{array}$ \\
\hline 2005 & Hovda KE, et al. & 51 & $\begin{array}{l}\text { Respiratory arrest, coma on presentation, severe } \\
\text { metabolic acidosis }(\mathrm{pH}<6.9, \mathrm{BD}>28) \text {, and } \\
\text { patients' inability to hyperventilate [8] }\end{array}$ \\
\hline 2007 & Paasma R, et al. & 111 & $\begin{array}{l}\text { Coma on presentation, degree of metabolic } \\
\text { acidosis, high serum methanol concentration and } \\
\text { the patients' inability to hyperventilate [24] }\end{array}$ \\
\hline 2007 & $\begin{array}{l}\text { Hassanian- } \\
\text { Moghaddam } \mathrm{H} \text {, et al. }\end{array}$ & 25 & $\begin{array}{l}\mathrm{pH}<7 \text {, coma on admission and }>24 \text { h of delay } \\
\text { from intake to admission }[25]\end{array}$ \\
\hline
\end{tabular}

normal or low BMI. The data of the enrolled patients was recorded in a standard questionnaire, designed before the study. The data included demographic information, clinical manifestations, time elapsed between ingestion and presentation, blood glucose level on presentation (before treatment), results of arterial blood gas analysis, and the clinical outcome. This questionnaire was filled out on the basis of the hospital charts, files, and autopsy reports registered in the forensic medical center of Tehran, and information was obtained by contacting the patients or relatives of the dead patients by telephone.

Statistical analysis was done using SPSS software (version 17, Chicago, Illinois, USA), and application of MannWhitney $U$ test, Pearson's chi-square test, Pearson correlation coefficient $(r)$, receiver operating characteristic (ROC) curve, and logistic regression. $P$ values less than 0.05 were considered to be statistically significant. Our study was approved by the Regional Ethics Committee.

\section{Results}

Ninety-five patients with methanol poisoning met the inclusion criteria and were included in the study. Of these, $91(96 \%)$ were male and $4(4 \%)$ were female. Mean age was $31.6 \pm 14.3$ years (range, 13 to 75 ) (Fig. 1). A total of 68 patients survived (72\%) and 27 expired (28\%). Comparisons between the admission data of the two groups (non-survivors and survivors) are shown in the Table 2. The median blood glucose level was $144 \mathrm{mg} / \mathrm{dL}$ (range, 75 to 500). There was no significant statistical correlation between blood glucose level and time to treatment $(r=-0.038$, $P=0.733)$, age $(r=0.154, P=0.136), \mathrm{pCO}_{2} \quad(r=0.133$,
$P=0.207)$, or serum bicarbonate concentration ( $r=-0.139, P=0.184)$, but blood glucose level had a significant statistical correlation with $\mathrm{pH}(r=-0.242$, $P=0.02)$ and base deficit (BD) $(r=0.230, P=0.03)$. Mean blood glucose level was $140 \pm 55$ and $219 \pm 99 \mathrm{mg} / \mathrm{dL}$ in the live and dead patients, respectively $(P<0.001)$. Considering the cutoff level of $140 \mathrm{mg} / \mathrm{dL}$ for blood glucose (obtained by ROC curve analysis), and adjusting according to the admission data

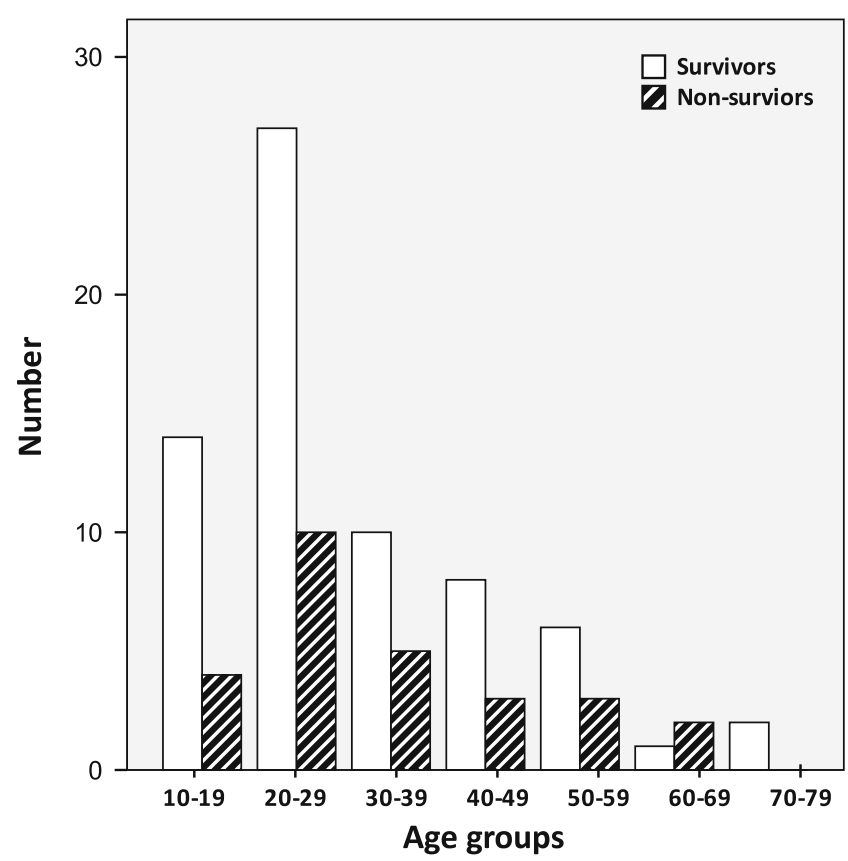

Fig. 1 Age of the survivor and non-survivor methanol-poisoned patients. There was no significant statistical difference between these two groups $(P=0.253)$ 
that had significant statistical differences between the two study groups using logistic regression analysis-i.e., elapsed time to treatment, coma, seizure, $\mathrm{pH}, \mathrm{pCO}_{2}$, serum bicarbonate concentration, and BD (Table 2) - the odds ratio for hyperglycemia (blood glucose levels more than $140 \mathrm{mg} / \mathrm{dL}$ ) as a risk factor for death was $6.5(95 \%$ confidence interval $(\mathrm{CI})=1.59$ 26.4).

\section{Discussion}

Poisoning with methanol is a classical example of a lifethreatening condition where species differences prevent us from obtaining information from the experimental studies [27]. Only certain primates and animals made folate deficient have been used as models [5, 28]. Other species do not develop acidosis or toxicity on exposure with methanol [1, 29]. Therefore, most of our knowledge is based on the case series and studies and outbreaks. It has been shown that prognosis for methanol poisoning depends on the degree of metabolic acidosis (reflected by the decrease in $\mathrm{pH}$ and increase in BD) $[1,8,17]$. Different findings on admission have been suggested as poor prognostic factors, e.g., high serum levels of methanol (not shown in this study) $[23,24,26]$ or the long time elapsed from consumption to the time of admission [22, 25]; however, others did not find these [8, 21, 25]. In methanol poisoning, coma on admission and seizures are generally considered to be poor prognostic signs $[8,21,23-$ 26]. Recently, respiratory arrest and increased $\mathrm{pCO}_{2}$ in the severely acidotic patients (lack of compensatory hyperventilation) have been suggested as new markers [8, 24].

The current study confirms earlier studies showing mortality correlating with coma and seizure on admission [8, 21, 23-26], severe metabolic acidosis [8, 21, 23-25], low serum bicarbonate levels [10], and a high base deficit [8] (Table 2). The current study also shows that hyperglycemia might be a new prognostic factor, as shown by regression analysis adjusted for other known prognostic factors $[8,10$, 21-26]. The odds ratio for hyperglycemia as a risk factor for death is here calculated to be $6.5(95 \% \mathrm{CI}=1.59-26.4)$.

The potential mechanism of hyperglycemia in methanol poisoning is not clear. It has been reported that methanol poisoning can be associated with acute pancreatitis $[12,30$, 31], and this can be suggestive of the role of acute pancreatitis in creating hyperglycemia. Hanston and Mahieu showed that acute pancreatitis in methanol poisoning might be more common than earlier thought. In their study, seven out of 22 patients were observed to have acute pancreatitis before treatment with ethanol [12]. Of course, regarding the current retrospective study which reviews the patients' files, the patients were not investigated at the time of hospitalization for biomarkers and the radiographic signs 
of pancreatitis. Also, the autopsy reports had not mentioned signs of pancreatitis and thus cannot be considered in our patients. In addition to that, few studies that reported the autopsy cases of methanol poisoning did not mention the pancreatic changes [32-34].

Another mechanism that may be suggestive of the increased blood glucose level in methanol poisoning is a stress-induced hyperglycemia, which is usually seen in the critically ill patients. In other words, increased counter regulatory hormones from the acute stress of methanol intoxication may contribute to hyperglycemia [35-38]. Therefore, it is possible that treating with insulin and glycemic control may have a role in the management of methanol poisoning. Further studies, particularly on the outbreak cases, are required to determine if this simple intervention may be of therapeutic value.

Interestingly, our study shows that there is a significant correlation between blood glucose level and both $\mathrm{pH}$ and $\mathrm{BD}$ : decrease in $\mathrm{pH}$ and increase in $\mathrm{BD}$ both seem to be correlated to an increase in the blood glucose level. Metabolic acidosis in methanol poisoning is either due to formate alone (early), or a combination of formate and lactic acidosis $[6,7]$. We have not found any known relation in the literature between hyperglycemia and the toxic mechanisms of formate and lactate, and so the mechanisms remain unknown $[1,8,17]$.

\section{Conclusions}

Our study shows that blood glucose levels were high in methanol poisoning. Hyperglycemia was significantly higher in those dying as compared to the survivors. Hyperglycemia might therefore be a new prognostic factor in methanol poisoning, but further studies are needed to determine whether controlling hyperglycemia may have therapeutic consequences.

\section{References}

1. Barceloux DG, Bond GR, Krenzelok EP, Cooper H, Vale JA, American Academy of Clinical Toxicology Ad Hoc Committee on the Treatment Guidelines for Methanol Poisoning (2002) American Academy of Clinical Toxicology practice guidelines on the treatment of methanol poisoning. J Toxicol Clin Toxicol 40 (4):415-446

2. Jacobsen D, McMartin KE (1986) Methanol and ethylene glycol poisonings. Mechanism of toxicity, clinical course, diagnosis and treatment. Med Toxicol 1(5):309-334

3. Sejersted OM, Jacobsen D, Ovrebø S, Jansen H (1983) Formate concentrations in plasma from patients poisoned with methanol. Acta Med Scand 213(2):105-110

4. Nicholls P (1976) The effect of formate on cytochrome aa3 and on electron transport in the intact respiratory chain. Biochim Biophys Acta 430(1):13-29
5. Herken W, Rietbrock N, Henschler D (1969) On the mechanism of methanol poisoning. The toxic agent and influence of acid-base balance. Arch Toxikol 24(2):214-238

6. Jacobsen D, McMartin KE (1997) Antidotes for methanol and ethylene glycol poisoning. J Toxicol Clin Toxicol 35(2):127-143

7. Hovda KE, Hunderi OH, Rudberg N, Froyshov S, Jacobsen D (2004) Anion and osmolal gaps in the diagnosis of methanol poisoning: clinical study in 28 patients. Intensive Care Med 30 (9): $1842-1846$

8. Hovda KE, Hunderi OH, Tafjord AB, Dunlop O, Rudberg N, Jacobsen D (2005) Methanol outbreak in Norway 2002-2004: epidemiology, clinical features and prognostic signs. J Intern Med 258(2):181-190

9. Teo SK, Lo KL, Tey BH (1996) Mass methanol poisoning: a clinico-biochemical analysis of 10 cases. Singapore Med J 37 (5):485-487

10. Bennet IL Jr, Cary FH, Mitchell GL Jr, Cooper MN (1953) Acute methyl alcohol poisoning: a review based on experiences in an outbreak of 323 cases. Medicine 32:431-463

11. Sefidbakht S, Rasekhi AR, Kamali K, Borhani Haghighi A, Salooti A, Meshksar A, Abbasi HR, Moghadami M, Nabavizadeh SA (2007) Methanol poisoning: acute MR and CT findings in nine patients. Neuroradiology 49(5):427-435

12. Hantson P, Mahieu P (2000) Pancreatic injury following acute methanol poisoning. J Toxicol Clin Toxicol 38(3):297-303

13. Roe O (1955) The metabolism and toxicity of methanol. Pharmacol Rev 7(3):399-412

14. Mégarbane B, Borron SW, Baud FJ (2005) Current recommendations for treatment of severe toxic alcohol poisonings. Intensive Care Med 31(2):189-195

15. Mégarbane B, Borron SW, Trout H, Hantson P, Jaeger A, Krencker E, Bismuth C, Baud FJ (2001) Treatment of acute methanol poisoning with fomepizole. Intensive Care Med 27 (8): $1370-1378$

16. Brent J, McMartin K, Phillips S, Aaron C, Kulig K (2001) Fomepizole for the treatment of methanol poisoning. $\mathrm{N}$ Engl $\mathrm{J}$ Med 344(6):424-429

17. Jacobsen D, Jansen H, Wiik-Larsen E, Bredesen JE, Halvorsen S (1982) Studies on methanol poisoning. Acta Med Scand 212(12):5-10

18. Jacobsen D, Ovrebø S, Sejersted OM (1983) Toxicokinetics of formate during hemodialysis. Acta Med Scand 214(5):409-412

19. Hovda KE, Froyshov S, Gudmundsdottir H, Rudberg N, Jacobsen $\mathrm{D}$ (2005) Fomepizole may change indication for hemodialysis in methanol poisoning: prospective study in seven cases. Clin Nephrol 64(3):190-197

20. Osterloh JD, Pond SM, Grady S, Becker CE (1986) Serum formate concentrations in methanol intoxication as a criterion for hemodialysis. Ann Intern Med 104(2):200-203

21. Naraqi S, Dethlefs RF, Slobodniuk RA, Sairere JS (1979) An outbreak of acute methyl alcohol intoxication. Aust N Z J Med 9 (1):65-68

22. Mathieu P, Hassoun A, Lauwerys R (1989) Predictors of methanol intoxication with unfavourable outcome. Hum Toxicol 8(2):135137

23. Liu JJ, Daya MR, Carrasquillo O, Kales SN (1998) Prognostic factors in patients with methanol poisoning. J Toxicol Clin Toxicol 36(3):175-181

24. Paasma R, Hovda KE, Tikkerberi A, Jacobsen D (2007) Methanol mass poisoning in Estonia: outbreak in 154 patients. Clin Toxicol Phila 45(2):152-157

25. Hassanian-Moghaddam H, Pajoumand A, Dadgar SM, Shadnia Sh (2007) Prognostic factors in methanol poisoning. Hum Exp Toxicol 26(7):583-586

26. Swartz RD, Millman RP, Billi JE, Bondar NP, Migdal SD, Simonian SK, Monforte JR, McDonald FD, Harness JK, Cole KL 
(1981) Epidemic methanol poisoning: clinical and biochemical analysis of a recent episode. Med Baltim 60(5):373-382

27. Roe O (1982) Species differences in methanol poisoning. Crit Rev Toxicol 10(4):275-286

28. Noker PE, Tephly TR (1980) The role of folates in methanol toxicity. Adv Exp Med Biol 132:305-315

29. McMartin KE, Martin-Amat G, Makar AB, Tephly TR (1977) Methanol poisoning. V. Role of formate metabolism in the monkey. J Pharmacol Exp Ther 201(3):564-572

30. Bennett IL Jr, Nation TC, Olley JF (1952) Pancreatitis in methyl alcohol poisoning. J Lab Clin Med 40(3):405-409

31. Eckfeldt JH, Kershaw MJ (1986) Hyperamylasemia following methyl alcohol intoxication. Source and significance. Arch Intern Med 146(1):193-194

32. Abdallat IM, Hadidi MS, Rashid N, Hadidi KA (2009) Outbreak of methanol fatalities in Jordan. J Bahrain Med Soc 21(3):283286
33. Andresen H, Schmoldt H, Matschke J, Flachskampf FA, Turk EE (2008) Fatal methanol intoxication with different survival timesmorphological findings and postmortem methanol distribution. Forensic Sci Int 179(2-3):206-210

34. Mittal BV, Desai AP, Khade KR (1991) Methyl alcohol poisoning: an autopsy study of 28 cases. J Postgrad Med 37(1):9-13

35. Lazzeri C, Tarquini R, Giunta F, Gensini GF (2009) Glucose dysmetabolism and prognosis in critical illness. Intern Emerg Med 4(2):147-156

36. McCowen KC, Malhotra A, Bistrian BR (2001) Stress-induced hyperglycemia. Crit Care Clin 17(1):107-124

37. Halter JB, Beard JC, Porte D Jr (1984) Islet function and stress hyperglycemia: plasma glucose and epinephrine interaction. Am J Physiol 247(1 Pt 1):E47-E52

38. Kajbaf F, Mojtahedzadeh M, Abdollahi M (2007) Mechanisms underlying stress-induced hyperglycemia in critically ill patients. Therapy 4:97-106 